\title{
Automatic Left Atrium Segmentation by Cutting the Blood Pool at Narrowings
}

\author{
Matthias John and Norbert Rahn \\ Siemens Medical Solutions, Henkestrasse 127, 91052 Erlangen,Germany \\ \{matthias.mj.john, norbert.rahn\} asiemens.de
}

\begin{abstract}
This paper presents a method to extract heart structures from CTA and MRA data sets, in particular the left atrium. First, the segmented blood pool is subdivided at narrowings in small components. Second, these basic components are merged automatically so that they represent the different heart structures. The resulting cutting surfaces have a relatively small diameter compared to the diameter of the neighboring heart chambers. Both steps are controlled by only one fixed parameter. The method is fast and allows interactive postprocessing by the user. Experiments on various data sets show the accuracy, robustness and repeatability of this approach.
\end{abstract}

\section{Introduction}

Atrial fibrillation is the most common heart arrhythmia and greatly increases the risk of stroke. This irregular heart rhythm originates in the atrial heart chambers. Over 2 million people are affected in the U.S. alone. One way to treat and cure atrial fibrillation is catheter ablation. First electrical signals in the heart muscle are mapped and localized. One successful approach to finally eliminate the atrial fibrillation is to isolate the pulmonary veins of the left atrium electrically from the rest of the heart by ablation. The dissimilarity of different left atrium shapes complicates the procedures. CT or MR imaging supports planning and intervention of these catheter ablations. After segmenting the left atrium and the pulmonary veins from the images the individual patient morphology can be visualized and the extracted boundary surface can be provided to electro-anatomical mapping systems.

CTA and MRA images are CT and MR images enhanced by contrast agent filled blood vessels and chambers. This provides higher image intensities for the blood pool than for surrounding tissues and helps to extract the left atrium voxels. Nevertheless, the left atrium segmentation is usually done manually or by segmentation tools that are not specific to this task, which yields segmentation times larger than 30 minutes. The reason for the absence of more specific automatic segmentation algorithms might be the complex and varying shape of the left atrium. (For instance position and number of the pulmonary veins can vary for different patients.) This is in contrast to the left ventricle, where a lot of segmentation approaches were suggested. Automatic methods for left ventricle segmentation are usually level-set methods [13], atlas-based methods or algorithms based on active shape models [3]. Active shape models are the most common approach for such segmentations. Recently, a shape model approach for the left atrium segmentation from CT images was proposed in [1]. 

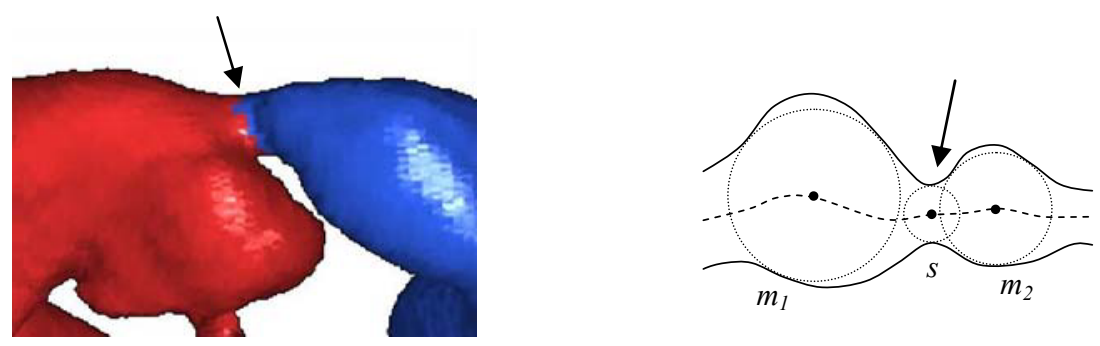

Fig. 1. Left: A narrowing and a possible cut. Right: Medial Axis (dashed line) of a two dimensional object (interior of the solid lines) and two locally maximal medial balls $m_{l}, m_{2}$ and the smallest medial ball $s$ between $m_{1}$ and $m_{2}$. The ball $s$ lies at the narrowing between $m_{1}$ and $m_{2}$.

In our method we make use of the fact that neighboring heart chambers can be separated with cuts at narrowings of the blood pool. The size of such a cutting surface is relatively small compared to the size of the neighboring heart chambers. We give an algorithmic formulation how to cut a blood pool at all narrowings. This subdivision into basic components is related to the medial axis transform which describes a volumetric object as a union of balls. The balls touch the surface of the object in at least two points, their center points build the medial axis (see Fig. 1). In this paper we associate with every locally maximal ball a basic component. If we follow the union of balls along the centers from a maximal ball to a neighboring maximal ball the radii first get smaller and then increase. At the smallest ball on this way we have a narrowing of the object, which is a possible cut. Because of this relation between our components and the medial axis transform we make use of a distance transformation algorithm [4] that helps to construct this data structure and gives information about the size of the basic components.

The merging of the basic components to build up the heart chambers is similar to the merging process used in watershed-transformation algorithms [11] and algorithms for surface reconstruction of geometric point sets [5]: In our setting two components are merged if the separating structure is large, so the difference to the size of the neighboring components is small. Observe that the common merging procedure for watershed-transformations in medical image segmentation handles every voxel as one element, whereas an element in our merging procedure is a basic component that usually consist of thousands of voxels. Furthermore the merging does not depend on the gray values but on the geometric structure of the object. Also for fast and efficient interactive post-processing by the user we adapt techniques originally developed for interactive watershed-transformations $[7,8]$.

\section{Method}

Before we discuss the technical details of our segmentation method we want to give an intuition about its basic ideas. Figure 2 gives an overview of the whole method.

To decompose a segmented blood pool object into the different heart chambers and vessel structures it can be cut at narrowings. Figure 1 shows such a narrowing for a vessel which might be a possible cut. The problem is to find the right cuts 


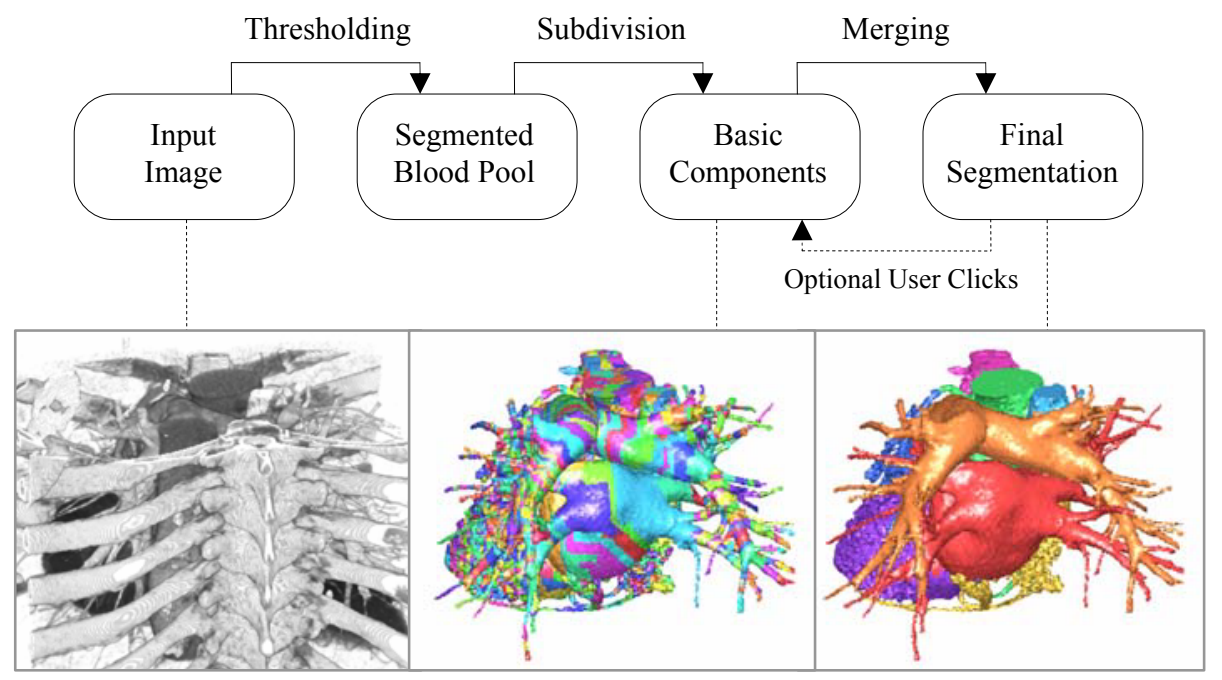

Fig. 2. Overview of the algorithm. Three steps of the algorithm are visualized in the images with a CTA data set. Left: A volume visualization of the complete data set. Middle: Subdivision of the blood pool into basic components. Right: Merged components that represent the heart chambers and vessels.

automatically. We can make the following observation: The diameter of the correct cuts is small compared to the diameter of the adjacent components.

The main idea of the algorithm is to subdivide the segmented blood pool object at all possible narrowings and merge the resulting basic components to get the heart structures. We start with the basic components and merge neighboring components if the diameter of a separating surface (i.e. the cut at the narrowing) is nearly as large as the diameter of the adjacent components. We end up with cuts with relatively small diameters compared to the adjacent components.

\subsection{Segmenting the Blood Pool}

The extraction of heart structures requires the extraction of the blood pool from the contrast enhanced CTA and MRA data sets. We use a simple threshold-based approach. With a given threshold we separate the blood pool voxels from other structures. This works fine with MRA data sets. With CTA data sets such a threshold also segments voxels that represent bone tissue. Fortunately, blood pool structures and bone structures only touch each other, but do not intersect deeply. Therefore, it is no problem to separate these structures by the subsequent steps of the algorithm (see Section 2.2 and 2.3).

For CTA data one can use a constant Hounsfield threshold for segmenting the blood pool. Alternatively, the algorithm can let the user pick a voxel inside the blood pool. A threshold can then be computed automatically from the local neighborhood of this seed point. 
If we allow the algorithm to let the user select a voxel inside the blood pool, we can use region growing. This helps to eliminate a lot of bone tissue voxels at the beginning and speeds up the subsequent algorithm steps.

\subsection{Computing Basic Components}

In this section we will show how to subdivide a binary image into several components. Figure 3 gives an overview of this part of the algorithm.

The first step is to compute the distance transformation of the binary image. For each marked voxel (inside object) we compute its squared Euclidian distance to the closest unmarked voxel (outside object). So voxels deep inside the object get a high value, voxels close to the boundary of the object get a small value. Zero is assigned to all voxels outside the object. Several algorithms were proposed for this task, see [4] for an overview. We use the fast and exact method described in [12].

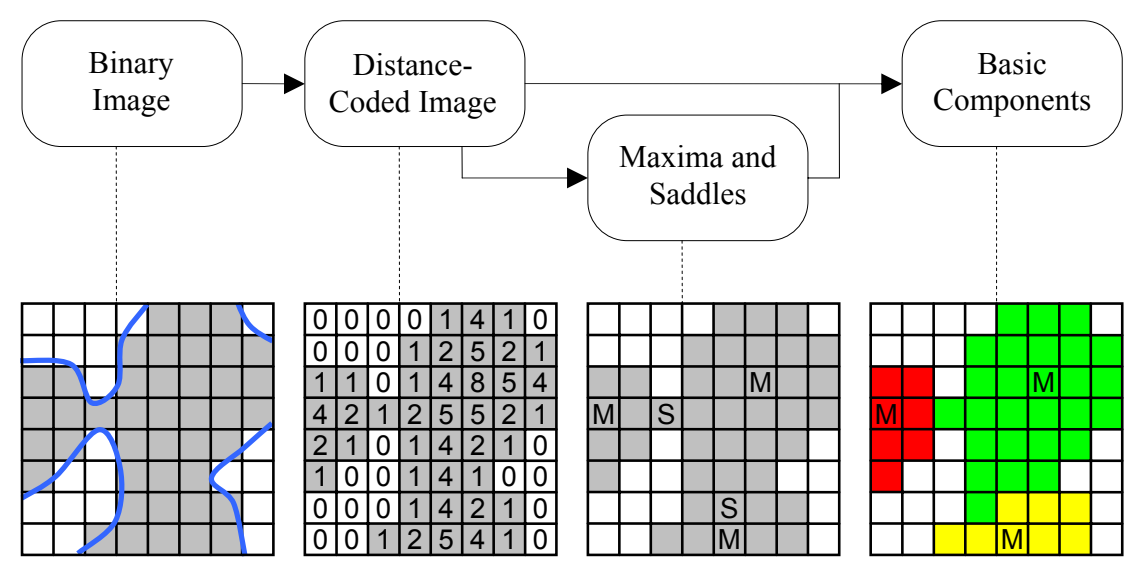

Fig. 3. Subdivision overview. The lower row shows a two dimensional example on a $2 \mathrm{D}$ grid. First, a distance transformation is computed. Then maxima and saddle points are extracted. Finally every object voxel is assigned to a maximum which results in the basic components.
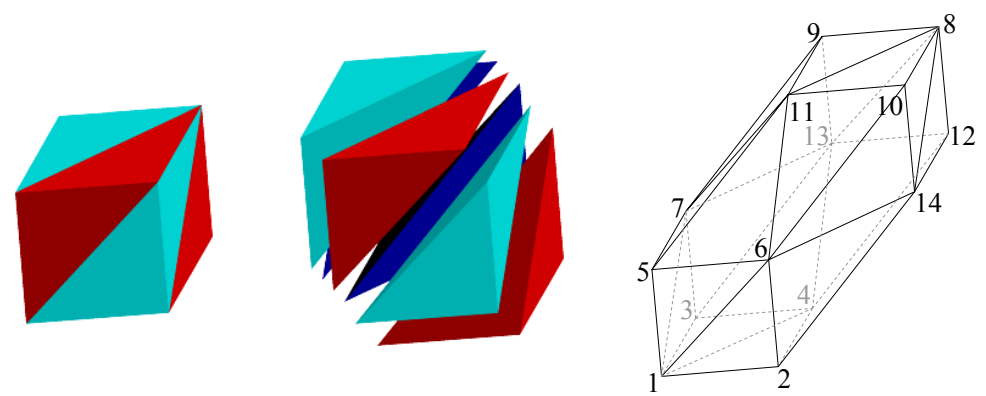

Fig. 4. Left: A Freudenthal subdivision of a cube. Right: The 14-neighborhood of a grid vertex of a regular cubic grid, if every grid cube is subdivided by the Freudenthal scheme. 
In a second step we determine all voxels that are local maxima and saddle points of the distance transformation. A (local) maximum is a voxel, that has a larger value than all its neighboring voxels. A saddle (point) is a voxel that has (at least) two voxels with larger values in its neighborhood and a ring of voxels with smaller values that separates the two larger ones.

Both maxima and saddles lie on the medial axis of the object. Every maximum lies in the center of a basic component. Every saddle lies at the center of a separating surface between two neighboring basic components which is the cutting surface at a narrowing of the object. The other way around, every basic component contains a maximum and every separating surface between two basic components contains a saddle. So, determining these two types of voxels helps to compute the basic components.

It can happen, that two neighboring voxels have the same value. To simplify the handling of maxima and saddles we want to avoid this situation when comparing voxels. Solutions to such equality problems are discussed in [11]. We prefer a simple one: We define a fixed order over all voxels (only depending on the $\mathrm{x}-, \mathrm{y}-$, and $\mathrm{z}-$ coordinates). In the case of equal voxel values we compare the voxels with respect to this order.

In a third step we assign every voxel to a basic component. Each local maximum is the center of a basic component. To assign a voxel to a basic component, we follow the gradient of this voxel until we end up in a maximum. To be more precise we follow a line of voxels with increasing distance function values until we reach a maximum. We determine this line by computing for each voxel the neighboring voxel with the steepest ascent.

The whole computation of maxima, saddles and basic components depends on the choice of the local neighborhood. We use a 14-neighborhood, i.e. 14 neighbors are assigned to each voxel (see Figure 4). This neighborhood comes from a tetrahedral subdivision of the regular cubic grid, where the grid vertices are the center points of the voxels. If we take a voxel $\mathrm{v}$, all voxels that are connected by an edge to $\mathrm{v}$ in the tetrahedral grid are defined as neighbors of $\mathrm{v}$. We get a regular 14-neighborhood if we use the Freudenthal subdivision. This and other tetrahedral subdivisions are discussed in [2]. The computation of maxima and saddles can be done fast using lookup tables; such techniques are described in [6] in the context of multiresolution isosurface extraction. The advantage of the 14-neighborhood is the resulting regular grid and the relatively small number of neighbors compared to the standard 26-neighborhood. Furthermore, we have the following guarantees: Every basic component contains a maximum and the surface between two components contains a saddle. This is because the 14-neighborhood goes back to a tetrahedral subdivision, which directly extends the discrete samples of the volume space to the whole space $\mathrm{R}^{3}$ by linear interpolation. So the maxima and saddles are critical points of a real continuous space and the guarantees described above come from the Morse theory of continuous spaces [10].

\subsection{Merging of Basic Components}

The granularity of the basic components is too large to represent real heart structures. Therefore we need to merge the basic components to build up larger structures. We start merging neighboring components $m_{1}$ and $m_{2}$ that have a separating surface $s$ with a large diameter, i.e. if 


$$
\text { merging value }\left(m_{1}, m_{2}, s\right)=\min \left(\operatorname{diam}\left(m_{1}\right), \operatorname{diam}\left(m_{2}\right)\right)-\operatorname{diam}(s)
$$

is small. The diameter of a component is the Euclidian distance function value of its corresponding maximum. The diameter of a separating surface is the Euclidian distance function value of its corresponding saddle.

Merging two components means algorithmically: Deleting symbolically the corresponding saddle and the smaller maximum and rearranging the saddles that were incident to the smaller maximum. Observe that some of the other merging values can change in this step.

We merge all neighboring components with a merging value smaller than a given threshold. We start with the components with the smallest merging value and go on in increasing order.

After the merging process the resulting components are separated at narrowings which have a small diameter compared to the diameter of the adjacent regions. Their merging value is larger than the given merging threshold.

\subsection{Interactive User Control}

After the previous algorithm steps the user can mark voxels as positive (= has to be part of the final segmentation) and negative (= not part of the final segmentation). We assign these markers to the basic components corresponding to the marked voxels. Now we can restart the merging process of Section 2.3, but we forbid merging of different labeled components and force merging of components with the same label.

Because the merging is very fast and the basic component subdivision can be reused, marking voxels by the user gives the user immediately a result.
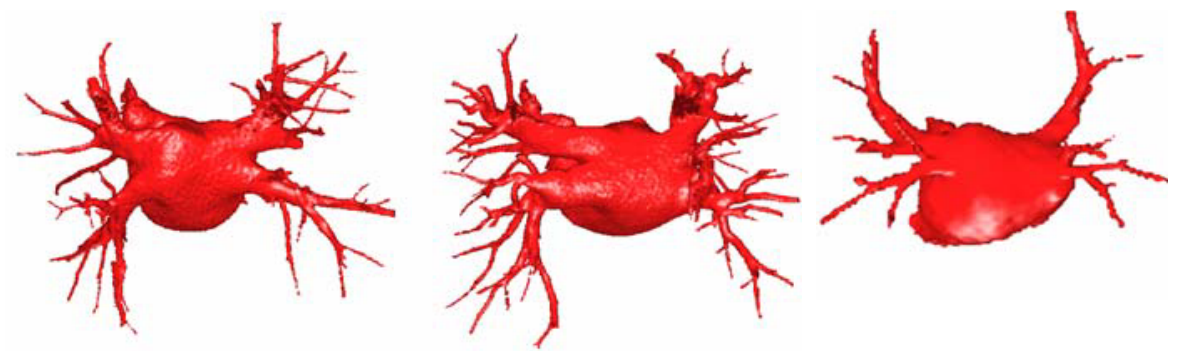

Fig. 5. Some results of the left atrium segmentation method. Left, Middle: CTA Right: MRA.

\section{Experiments and Results}

We applied our algorithm to 40 coronary CTA and 20 coronary MRA data sets from different manufacturers and clinics. The results were evaluated and discussed with electrophysiologists and radiologists. The goal of our study was to extract the left atrium with as little user interaction as possible. The user started the segmentation by selecting a voxel in the left atrium as a seed point. After the automatic segmentation procedure the user was able to mark additional voxels to interactively improve the result. The results were visualized either by a marching cubes isosurface [9] or by volume rendering. 
All data sets had an in-slice resolution of $512 \times 512$ voxels, the number of slices varied from 40 to 500 , and the voxel size varied from $0.318 \times 0.318 \times 0.4 \mathrm{~mm}^{3}$ to $0.859 \times 0.859 \times 1.4 \mathrm{~mm}^{3}$. The data sets included anomaly cases (more than two pulmonary veins on one side of the left atrium) and images with rather bad scan quality.

In all data sets we successfully extracted the left atrium with a merging threshold of $2.3 \mathrm{~mm}$. On some of the data sets user interaction helped to improve the results, usually for images with poor quality. In all cases the algorithm was able to segment the left atrium including all pulmonary veins and the left atrial appendage. All neighboring structures like the left ventricle, the right atrium, and veins close to the pulmonary veins were excluded. Furthermore, the bone structures in the CTA images were eliminated correctly. The segmentation results were practically independent of the exact position of the users click into the left atrium. This is mainly due to the computation and merging of the basic components which only depends on the geometry of the blood pool. Noisy data sets did not much effected the merging results, because small components due to noise are merged in an early phase to larger and more robust components.

We tested the algorithm on a $2 \mathrm{GHz}$ Pentium IV PC with $1 \mathrm{~GB}$ of RAM. The runtime of the algorithm depends on the number of blood pool voxels and varies between 5 (for small MRA data sets) and 45 seconds (for large CTA data sets). After the user added an additional marker the update of the segmentation took always less than a second, which made interactive post-processing with immediate feedback feasible.

\section{Conclusions}

To extract the left atrium from CTA and MRA images we suggest to cut the blood pool using geometric properties, i.e. to cut at narrowings that are small compared to their neighboring components. This approach gives correct results, which was tested on $40 \mathrm{CTA}$ and $20 \mathrm{MRA}$ data sets. The results are repeatable because of the stable method and the minimization of user interaction. The algorithm is fast, especially the merging procedure (that operates on the set of maxima and saddles which is much smaller than the number of all object voxels). The algorithm gives robust results even for noisy data sets.

To further evaluate the algorithm we will start a more detailed clinical evaluation. Besides supporting the intervention by visualizing the left atrium we will use the results for accurate $3 \mathrm{D}$ volume measurements and the support of electro-anatomical mapping systems. Possible future research directions are the automatic segmentation of other heart chambers and the automatic bone removal from CTA images.

\section{References}

1. Berg, J. v., Lorenz, C.: Accurate left atrium segmentation in multislice CT images using a shape model. Proc. SPIE Medical Imaging, San Diego, 2005.

2. Carr, H., Möller, T. Snoeyink, J.: Simplicial subdivisions and sampling artifacts. Proc. $11^{\text {th }}$ IEEE Visualization, 99-106, 2001.

3. Cootes, T. F., Taylor, C. J.: Statistical models of appearance for medical image analysis and computer vision. Proc. SPIE Medical Imaging, San Diego, 2001. 
4. Cuisenaire O.: Distance transformations: fast algorithms and applications to medical image processing. PhD thesis, Université catholique de Louvain (UCL), Belgium, 1999.

5. Giesen, J., John, M.: Surface reconstruction based on a dynamical system. Computer Graphics Forum, 21(3):363-371, 2002.

6. Gerstner, T., Pajorola, R.: Topology preserving and controlled topology simplifying multiresolution isosurface extraction. Proc. $10^{\text {th }}$ IEEE Visualization , 259-266, 2000.

7. Kahn, H. K., Peitgen, H. O., IWT - Interactive watershed transform: A hierarchical method for efficient interactive and automated segmentation of multidimensional grayscale images. Proc. SPIE Medical Imaging, 5032:643-653, 2003.

8. Kuhnigk, J. M., Hahn, H. K., Hindennach, M., Dicken, V., Krass, S., Peitgen, H. O.: Lung lobe segmentation by anatomy-guided 3D watershed transform. Proc. SPIE Medical Imaging, 5032:1482-1490, 2003.

9. Lorensen, W., Cline, H.: Marching cubes: A high resolution 3D surface construction algorithm. Computer Graphics, 21(4): 163-169, 1987.

10. Milnor, J.: Morse Theory. Princeton University Press, New Jersey, 1963.

11. Roerdink, J. B. T. M., Meijster, A.: The watershed transform: Definitions, algorithms, and parallelization strategies, Fundamenta Informaticae, 41:187-228, 2000.

12. Saito, T., Toriwaki, J.: New algorithms for Euclidian distance transformation of an ndimensional digitized picture with applications. Pattern Recognition, 27(11):1551-1565, 1994.

13. Sethian, J. A.. Level set methods and fast marching methods: Evolving interfaces in computational geometry, fluid mechanics, computer vision, and materials science. Cambridge University Press, 1999. 